\title{
ESTUDO PROSPECTIVO DOS EFEITOS DA AMIODARONA NA FUNÇÃO TIROIDIANA DE PACIENTES CHAGÁSICOS EM ÁREA DE DEFICIÊNCIA DE IODO
}

\author{
Maria Aparecida Enes de Barros e Rui Monteiro de Barros Maciel
}

\begin{abstract}
Com o objetivo de avaliar a função tiroidiana após uso crônico da amiodarona, em área de deficiência de iodo e endemia chagásica, 24 pacientes foram analisados antes e após três e nove meses de uso da droga. A avaliação constou de exame clínico, dosagem sérica de T4, T3, rT3, TSH, anticorpo antitiroglobulina e TSH 30 minutos após infusão venosa de uma ampola de $200 \mu \mathrm{g}$ de TRH. A captação do iodo radioativo 131 e a cintilografia da tiróide foram realizadas antes e aos 9 meses após tratamento. Disfunção tiroidiana ocorreu em 20,8\% dos pacientes sendo $12,5 \%$ de hipertiroidismo e $8,3 \%$ de hipotiroidismo, com anticorpos antitiroglobulina negativos. Captação do iodo radioativo 131 foi positiva em um paciente hipertiroideo com bócio. O diagnóstico de hipertiroidismo foi melhor evidenciado pela resposta reduzida ou bloqueada do TSH ao TRH e não pela concentração do T3 no soro e o de hipotiroidismo pela concentração elevada do TSH. O TSH elevado desde o início do tratamento pode predispor ao aparecimento de bócio. Concluimos que o uso da amiodarona em nossa regiäodeve ser judiciosamente analisado, sendo a função tiroidiana cuidadosamente monitorizada antes e durante o tratamento.
\end{abstract}

Palavras-chaves: Amiodarona. Função tiroidiana. Doençade Chagas. Deficiência de iodo.

A amiodarona, droga que contém dois átomos de iodo, bloqueia a conversão periférica de $\mathrm{T} 4 \mathrm{em}$ $\mathrm{T} 3^{523}$, pela inibição da enzima hepatica 5 '-desiodase (5'-D), com diminuição do T3 e aumento do rT3 e do T4 séricos ${ }^{6}$. Também inibe a enzima $5^{\prime}$-D tipo II da hipófise ${ }^{26}$, a qual converte $\mathrm{T} 4$ em $\mathrm{T}^{29}$ o que pode também ser atribuído a niveis elevados de $\mathrm{rT}^{30}$. Seu uso crônico produz elevada incidência de disfunção tiroidiana ${ }^{14} 1727$, sendo o hipertiroidismo mais relatado emnáreas geográficas de deficiência de iodo ${ }^{173}$ e o hipotiroidismo em área de suficiência de iodo ${ }^{17}$. Além disto, a droga pode causar lesões foliculares degenerativas destrutivas e fibrose na tiróide ${ }^{32}$. Mecanismos imunológicos têm sido sugeridos como indutores do hipertiroidismo ${ }^{24}$, com alteração da função das células $T$ pela amiodarona. No hipotiroidismo a captação do iodo radioativo $\left({ }^{131} \mathrm{I}\right)$ pode estar diminuída ${ }^{8}$ ou preservada ${ }^{35}$ pelo excesso de ic deto.

\footnotetext{
Curso de Pós-Graduação em Endocrinologia da Faculdade de Medicina da Escola Paulista de Medicina, São Paulo, SF e Faculdade de Medicina do Triângulo Mineiro, Uberaba, MG. Endereço para correspondência: Prof ${ }^{\mathbf{a}}$. Maria Aparecida Enes Barros. Depto. de Medicina, Endocrinologia/FMTM. Av. Getúlio Guaritá s/n, 38025-050 Uberaba, MG.

Recebido para publicação em 28/02/94.
}

Trabalhando no Triângulo Mineiro, região considerada de insuficiência iódica ${ }^{2021}$ e onde há grande número de cardiopatas chagásicos ${ }^{15}$, muitos usando amiodarona, decidimos estudar a função tiroidiana após o uso crônico do referido antiarrítmico.

\section{MATERIAL E MÉTODOS}

Estudamos 24 pacientes provenientes dos Ambulatórios de Cardiologia e Endocrinologia do Departamento de Clínica Médica da Faculdade de Medicina do Triângulo Mineiro em Uberaba, Minas Gerais (Tabela 1). Todos eram portadores de arritmia cardíaca do tipo extra-sistolia ventricular e tinham reação reagente de imunofluorescência para Trypanosoma cruzi. Nenhum paciente havia usado amiodarona previamente.

Avaliamos a função tiroidiana antes do uso da amiodarona por meio das dosagens hormonais do T4 (4,5 a $12 \mu \mathrm{g} / \mathrm{dl})$, T3 (72 a $214 \mathrm{ng} / \mathrm{dl})$, rT3 (2 a $34 \mathrm{ng} / \mathrm{dl})$, TSH ( 1 a $5 \mathrm{mU} / \mathrm{L})$ por radioimunoensaio ${ }^{16}$ 25 e dos anticorpos antitiroglobulina (ACAT) por hemaglutinação passiva ${ }^{3}$ e enzima-imunoensaio ${ }^{12}$. O TSH foi novamente dosado 30 minutos após infusão venosa de uma ampola de TRH $(200 \mu \mathrm{g})$. Os 
Barros MAE, Maciel RMB. Estudo prospectivo dos efeitos da amiodarona na função tiroidiana de pacientes chagásicos em área de deficiência de iodo. Revista da Saciedade Brasileira de Medicina Tropical 27:149-155, jul-set, 1994.

Tabela 1 - Dados de identificação de 24 pacientes com reação de imunofluorescência para doença de Chagas reagente.

\begin{tabular}{cccc}
\hline Caso & Sexo & Idade (anos) & Cor \\
\hline 1 & M & 25 & P \\
2 & M & 51 & B \\
3 & F & 44 & B \\
4 & F & 42 & B \\
5 & F & 33 & B \\
6 & M & 53 & B \\
7 & F & 50 & B \\
8 & F & 57 & B \\
9 & F & 46 & P \\
10 & F & 76 & B \\
11 & M & 18 & B \\
12 & M & 47 & P \\
13 & F & 57 & P \\
14 & F & 72 & B \\
15 & F & 50 & B \\
16 & F & 68 & B \\
17 & F & 44 & B \\
18 & F & 36 & B \\
19 & F & 74 & B \\
20 & F & 45 & B \\
21 & F & 56 & P \\
22 & F & 62 & B \\
23 & F & 38 & B \\
24 & F & 47 & B \\
\hline
\end{tabular}

M: masculino

F: feminino

P: preta

B: branca

pacientes passaram então a receber amiodarona (600mg na primeira semana e 200 a $400 \mathrm{mg}$ a seguir). Repetimos a avaliação da função tiroidiana três e nove meses após o início do tratamento, do mesmo modo como havíamos feito antes.

A captação do iodo radioativo e o mapeamento da tiróide foram realizados no tempo zero (basal) e após nove meses de uso da amiodarona, utilizandose, para tal procedimento, um mapeador linear.

Para o diagnóstico de hipertiroidismo levamos em consideração sinais e sintomas de tirotoxicose, aumento de T4 sérico, aumento de T3 sérico, e resposta bloqueada do TSH ao TRH. Hipotiroidismo foi diagnosticado pela elevação sérica da concentração de TSH e diminuição abaixo do nível da normalidade do $\mathrm{T} 4$, com ou sem sinais e sintomas de hipotiroidismo.

Os dados numéricos foram submetidos à análise estatística através do teste de Friedman ${ }^{28}$, MannWhitney e pelo coeficiente de correlação de
Spearman ${ }^{28}$. Para fins de conclusões em termos estatísticos fixamos em todos os testes o nível de significância de 0,05 .

\section{RESULTADOS}

Nenhum paciente apresentou evidência clínica ou laboratorial de doença tiroidiana antes do uso da amiodarona. Após o tratamento, dos 24 pacientes, $18(75 \%)$ tinham elevação dos níveis séricos de T4, dez em ambas as avaliações, dois no $3^{\circ}$ mês e seis no $9^{\circ}$ mês (Tabela 2). Quatro $(16,6 \%)$ pacientes não mostraram alteração após o tratamento. Em conjunto, a mediana, três meses após a terapêutica, apresentou aumento de $19,6 \%$ e aos nove meses 27,4\% (Tabela 4). Após o uso da amiodarona, dos 24 pacientes, três $(12,5 \%)$ foram considerados como tendo adquirido hipertiroidismo (casos 15 , 19 e 22), e dois $(8,3 \%$ ) de hipotiroidismo (casos 3 e 16) (Tabela 2). Ambos apresentaram os sinais e sintomas de hipofunção glandular e valor de T4 abaixo do limite inferior da normalidade no $9^{\circ}$ mês de tratamento.

Quanto ao T3 sérico, dos 24 pacientes estudados, nove $(37,5 \%)$ apresentaram valores diminuídos em algum momento do seguimento, sem quadro clínico de hipotiroidismo, sendo dois em ambas as avaliações, cinco só no $3^{\circ}$ mês e dois apenas no $9^{\circ}$ mês de tratamento (Tabela 2). Quinze $(62,5 \%)$ pacientes não mostraram anormalidade após tratamento. A mediana três meses após o tratamento mostrou diminuição de $25,3 \%$ e aos nove meses 13,0\% (Tabela 3).

Dos três pacientes que evoluíram para hipertiroidismo, dois tiveram redução dos seus valores iniciais de T3 aos três e aos nove meses. Dos dois pacientes hipotiroidianos o caso 16 mostrou nível de T3 abaixo da faixa da normalidade aos nove meses.

O valor basal de rT3 estava acima da faixa considerada normal para o método em $16(66,6 \%)$ dos 24 pacientes (Tabela 2). Aos três meses, 22 apresentaram valores de rT3 acima dos níveis superiores da normalidade aos três meses $(91,6 \%)$ e 23 $(95,8 \%$ ) aos nove meses (Tabela 2). A mediana três meses após o tratamento mostrou aumento de $78,3 \%$ e aos nove meses $62,1 \%$ e tais valores são estatisticamente significantes (Tabela 4).

Três dos 24 pacientes, incluindo os que 
Barros MAE, Maciel RMB. Estudo prospectivo dos efeitos da amiodarona na função tiroidiana de pacientes chagásicos em área de deficiência de iodo. Revista da Sociedade Brasileira de Medicina Tropical 27:149-155, jul-set, 1994.

Tabela 2 - Valores individuais do T4 (ug/dl), T3 (ng/dl), rT3 (ng/dl) em chagásicos, antes e aos três e nove meses após tratamento com amiodarona.

\begin{tabular}{|c|c|c|c|c|c|c|c|c|c|}
\hline \multirow[t]{2}{*}{ Caso } & \multicolumn{3}{|c|}{ Inicial } & \multicolumn{3}{|c|}{3 meses } & \multicolumn{3}{|c|}{9 meses } \\
\hline & $\mathrm{T} 4$ & T3 & rT3 & $\mathrm{T} 4$ & $\mathrm{~T} 3$ & rT3 & $\mathrm{T} 4$ & $\mathrm{~T} 3$ & rT3 \\
\hline 1 & 7,0 & 127 & 35 & 8,0 & 68 & 48 & 14,5 & 72 & 60 \\
\hline 2 & 10,2 & 171 & 36 & 11,2 & 203 & 37 & 11,0 & 170 & 38 \\
\hline 3 & 10,8 & 153 & 37 & 10,2 & 95 & 89 & 4,0 & 80 & 58 \\
\hline 4 & 8,5 & 147 & 30 & 13,0 & 64 & 63 & 10,0 & 113 & 70 \\
\hline 5 & 12,0 & 170 & 27 & 18,0 & 157 & 89 & 13,0 & 162 & 60 \\
\hline 6 & 9,1 & 148 & 56 & 10,2 & 50 & 58 & 13,0 & 64 & 60 \\
\hline 7 & 11,1 & 151 & 43 & 9,0 & 105 & 23 & 8,5 & 201 & 26 \\
\hline 8 & 7,7 & 130 & 57 & 11,5 & 51 & 75 & 11,5 & 50 & 80 \\
\hline 9 & 9,8 & 149 & 38 & 19,0 & 94 & 80 & 16,0 & 60 & 90 \\
\hline 10 & 11,1 & 92 & 64 & 11,8 & 97 & 65 & 13,0 & 122 & 113 \\
\hline 11 & 10,9 & 126 & 77 & 12,2 & 135 & 89 & 16,0 & 115 & 61 \\
\hline 12 & 10,7 & 187 & 35 & 11,0 & 67 & 91 & 14,0 & 85 & 175 \\
\hline 13 & 7,8 & 130 & 27 & 9,4 & 140 & 27 & 13,0 & 80 & 56 \\
\hline 14 & 11,5 & 135 & 39 & 11,2 & 136 & 86 & 11,5 & 132 & 43 \\
\hline 15 & 7,7 & 125 & 25 & 12,0 & 83 & 79 & 12,5 & 78 & 197 \\
\hline 16 & 12,0 & 113 & 35 & 6,0 & 83 & 66 & 4,0 & 70 & 54 \\
\hline 17 & 11,2 & 166 & 36 & 14,4 & 50 & 135 & 14,0 & 118 & 138 \\
\hline 18 & 9,5 & 116 & 27 & 15,0 & 65 & 171 & 12,0 & 75 & 44 \\
\hline 19 & 11,0 & 140 & 33 & 14,0 & 130 & 197 & 14,4 & 83 & 170 \\
\hline 20 & 9,5 & 110 & 44 & 13,5 & 211 & 62 & 17,0 & 164 & 43 \\
\hline 21 & 8,4 & 122 & 21 & 13,5 & 88 & 112 & 12,6 & 46 & 137 \\
\hline 22 & 11,7 & 112 & 50 & 17,0 & 159 & 64 & 13,1 & 89 & 35 \\
\hline 23 & 12,0 & 175 & 45 & 12,2 & 162 & 64 & 12,4 & 182 & 54 \\
\hline 24 & 10,0 & 113 & 31 & 14,0 & $\$ 12$ & 78 & 13,3 & 148 & 70 \\
\hline
\end{tabular}

evoluíram para hipotiroidismo, tinham valores de TSH acima da normalidade no $3^{\circ}$ mês e cinco no $9^{\circ}$ mês (Tabela 3), aliás, os valores de TSH foram significativamente maiores no $9^{\circ}$ mês de tratamento. Dos pacientes que evoluíram para hipertiroidismo, dois já apresentavam o TSH no limite inferior da detectabilidade no $3^{\circ}$ mês de tratamento. Houve elevação significante da média dos valores do TSH pós TRH, aos nove meses do tratamento com amiodarona (Tabela 4).

Os pacientes tirotóxicos com resposta ausente ou bloqueada do TSH ao TRH apresentaram valores do T4 aumentados (Tabela 3). Dos dois pacientes hipotiroidianos o caso 3 apresentou resposta exagerada do TSH ao TRH no $3^{\circ}$ e $9^{\circ}$ meses e o 16 somente no $9^{\circ}$ mês do tratamento, e inclusive exibiu bócio na fase final da pesquisa. Nenhum paciente que desenvolveu hipertiroidismo ou hipotiroidismo apresentou positividade dos anticorpos antitiroglobulina. Observamos que três pacientes (caso 1, 4 e 5) que apresentaram hiper- resposta do TSH ao TRH no $3^{\circ}$ mês, mantida em dois deles (caso 4 e 5) no $9^{\circ}$ mês, eram normais. Tåmbém, dois pacientes (caso 6 e 17) que aos nove meses mostraram hiper-resposta do TSH ao TRH não apresentaram disfunção tiroideana.

Nos 24 pacientes, a captação do ${ }^{131}$ I pela tiróide que antes do tratamento mostrou níveis normais (18 $\pm 3.1 \%)$ evidenciou queda, estatisticamente significante, após nove meses de tratamento $(3,3 \pm$ $3,7 \%$ ) (Tabela 5). Somente um $(4,1 \%$ ) (caso 22 ) apressentou captação maior que a inicial, tendo se tornado hipertiroideo com bócio difuso.

Nos 24 pacientes a cintilografia da tiróide mostrou mapeamento normal da glândula na fase inicial. Após nove meses o mapeamento foi positivo em três pacientes (caso 11, 17 e 22) sendo o caso 22 hipertiroidiano.

A dosagem de ACAT mostrou-se positiva em somente um paciente (caso 10) na fase inicial e aos nove meses após a droga. 
Barros MAE, Maciel RMB. Estudo prospectivo dos efeitos da amiodarona na função tiroidiana de pacientes chagásicos em área de deficiencia de iodo. Revista da Sociedade Brasileira de Medicina Tropical 27:149-155, jul-set, 1994.

Tabela 3 - Valores de TSH (mU/L) basal e pós TRH, em chagásicos, antes $e$ aos três e nove meses após uso da amiodarona.

\begin{tabular}{|c|c|c|c|c|c|c|}
\hline \multirow[t]{2}{*}{ Caso } & \multicolumn{2}{|c|}{ Inicial } & \multicolumn{2}{|c|}{3 meses } & \multicolumn{2}{|c|}{9 meses } \\
\hline & B & $\mathbf{P}$ & B & $\mathbf{P}$ & $\mathrm{B}$ & $\mathbf{P}$ \\
\hline 1 & 3,5 & 10,4 & 5,0 & 23,0 & 2,6 & 14,0 \\
\hline 2 & 1,9 & 8,7 & 3,5 & 9,5 & 5,3 & 10,0 \\
\hline 3 & 4,1 & 22,7 & 8,4 & 60,0 & 22,0 & 80,0 \\
\hline 4 & 3,0 & 20,0 & 7,1 & 22,0 & 5,9 & 24,0 \\
\hline 5 & 2,1 & 20,0 & 4,7 & 22,0 & 8,2 & 29,0 \\
\hline 6 & 3,3 & 21,0 & 2,6 & 20,0 & 4,7 & 22,0 \\
\hline 7 & 1,0 & 9,2 & 1,1 & 13,5 & 1,2 & 14,0 \\
\hline 8 & 1,4 & 5,4 & 1,0 & 7,8 & 1,4 & 7,5 \\
\hline 9 & 3,3 & 7,3 & 1,0 & 10,5 & 1,0 & 8,4 \\
\hline 10 & 1,6 & 30,0 & 1,3 & 15,0 & 2,1 & 17,0 \\
\hline 11 & 2,8 & 39,6 & 3,7 & 12,5 & 4,3 & 18,0 \\
\hline 12 & 3,2 & 8,2 & 3,5 & 20,0 & 3,4 & 15,0 \\
\hline 13 & 4,8 & 12,7 & 1,0 & 7,8 & 4,8 & 14,8 \\
\hline 14 & 1,0 & 10,0 & 2,3 & 15,8 & 2,1 & 14,0 \\
\hline 15 & 4,0 & 4,5 & 1,0 & 2,0 & 1,0 & 1,0 \\
\hline 16 & 3,0 & 9,4 & 13,0 & 16,0 & 30,0 & 30,0 \\
\hline 17 & 4,2 & 17,1 & 1,8 & 12,0 & 4,8 & 30,0 \\
\hline 18 & 1,6 & 8,2 & 1,8 & 17,0 & 3,8 & 12,5 \\
\hline 19 & 1,6 & 3,2 & 3,8 & 2,0 & 3,0 & 2,0 \\
\hline 20 & 2,1 & 15,0 & 2,4 & 16,0 & 1,9 & 18,0 \\
\hline 21 & 1,1 & 6,4 & 2,1 & 10,0 & 1,4 & 9,2 \\
\hline 22 & 1,0 & 4,6 & 1,0 & 4,8 & 2,0 & 1,0 \\
\hline 23 & 1,4 & 16,0 & 1,0 & 10,0 & 2,2 & 10,4 \\
\hline 24 & 1,0 & 10,0 & 1,0 & 8,2 & $-1,0$ & 4,1 \\
\hline
\end{tabular}

\section{DISCUSSÃO}

Nossos resultados comprovam a existência de disfunção tiroidiana com alterações dos hormônios tiroidianos em chagásicos tratados com amiodarona em região do Brasil carente de iodo. Um importante trabalho que conhecemos também em área de carência iodada foi realizado por Martino e cols ${ }^{17} \mathrm{em}$ Luca e Pisa, na Itália. Do ponto de vista qualitativo os resultados desses autores e os nossos são similares, o fato provavelmente se deve a quantidades farmacológicas de iodeto liberadas durante o metabolismo da amiodadora ${ }^{19}$. Do ponto de vista quantitativo nós obtivemos um percentual maior de disfunção tiroidiana $(20,8 \%)$ tanto hipertiroidismo $(12,5 \%)$ como hipotiroidismo $(8,3 \%)$. Os valores basais de RT3 foram bem mais elevados do que os encontrados por Martino e cols ${ }^{17}$. Aventamos três hipóteses que poderiam explicar tais diferenças: concomitância da doença de Chagas, área com maior deficiência de iodo e maior duração do tratamento com amiodarona. A importância da doença de Chagas na disfunção tiroidiana após amiodarona teria deser pesquisada através de estudo com controle não chagásicos. $O$ grau da deficiência de iodo, obviamente, deve influir na prevalência da disfunção tiroidiana.

Tabela 4 - Mediana dos valores dos hormônios tiroidianos (T3, T4 e rT3) antes e aos três e nove meses após tratamento com amiodarona.

\begin{tabular}{cccc}
\hline $\begin{array}{c}\text { Hormônios } \\
\text { tiroidianos }\end{array}$ & Antes & \multicolumn{2}{c}{ Após } \\
\cline { 3 - 4 } & $10,2(7,0-12,0)$ & $12,2(6,0-19,0)^{*}$ & $13,0(4,0-17,0)^{*}$ \\
\hline $\begin{array}{c}\mathrm{T} 4 \\
(\mu \mathrm{g} / \mathrm{dl}) \\
\mathrm{T} 3\end{array}$ & $130,0(92,0-187,0)$ & $97,0(50,0-211,0)^{*}$ & $113,0(46,0-201,0)^{*}$ \\
$(\mathrm{ng} / \mathrm{dl})$ \\
$\mathrm{rT3}$ \\
$\begin{array}{c}\mathrm{ng} / \mathrm{dl}) \\
\mathrm{TSH} \text { basal } \\
(\mathrm{mU} / \mathrm{L})\end{array}$ & $37,0(21,0-77,0)$ & $66,0(23,0-197,0)^{*}$ & $60,0(26,0-197,0)^{*}$ \\
$\begin{array}{c}\text { TSH pós TRH } \\
(\mathrm{mU} / \mathrm{L})\end{array}$ & $13,2(3,2-39,6)$ & $14,8(2,0-60,0)^{*}$ & $17,3(1,0-80,0)^{*}$ \\
\hline
\end{tabular}

* Teste de Friedman, com diferenças significatiyas em relação aos valores iniciais $(p<0.05)$. 
Barros MAE, Maciel RMB. Estudo prospectivo dos efeitos da amiodarona na funçäo tiroidiana de pacientes chagásicos em área de deficiência de iodo. Revista da Sociedade Brasileira de Medicina Tropical 27:149-155, jul-set, 1994.

Tabela 5 - Captação do lodo Radioativo 131 e cintilografia da tiróide, em chagásicos crônicos, antes e após nove meses de uso da amiodarona.

\begin{tabular}{|c|c|c|c|c|}
\hline \multirow[t]{2}{*}{ Caso } & \multicolumn{2}{|c|}{ Captação ${ }^{131}$ I (\% 24h) } & \multicolumn{2}{|c|}{ Cintilografia } \\
\hline & basal & 9 meses & basal & 9 meses \\
\hline 1 & 27,0 & 3,0 & $\mathrm{TN} *$ & - \\
\hline 2 & 17,0 & 3,0 & TN & - \\
\hline 3 & 17,5 & 2,5 & TN & - \\
\hline 4 & 19,0 & 2,0 & $\mathrm{TN}$ & - \\
\hline 5 & 15,0 & 2,0 & TN & - \\
\hline 6 & 19,0 & 2,0 & TN & - \\
\hline 7 & 17,0 & 2,5 & TN & - \\
\hline 8 & 20,0 & 2,0 & TN & - \\
\hline 9 & 14,0 & 2,8 & TN & - \\
\hline 10 & 14,5 & 3,5 & TN & - \\
\hline 11 & 20,0 & 5,5 & TN & + \\
\hline 12 & 20,0 & 1,5 & TN & - \\
\hline 13 & 16,0 & 3,0 & TN & - \\
\hline 14 & 17,0 & 0,0 & TN & - \\
\hline 15 & 18,0 & 2,0 & TN & - \\
\hline 16 & 16,0 & 3,0 & TN & - \\
\hline 17 & 15,0 & 4,5 & TN & + \\
\hline 18 & 20,0 & 3,0 & TN & - \\
\hline 19 & 15,0 & 2,0 & TN & - \\
\hline 20 & 14,0 & 4,0 & TN & - \\
\hline 21 & 17,0 & 1,7 & TN & - \\
\hline 22 & 17,0 & 20,0 & TN & + \\
\hline 23 & 19,0 & 2,0 & TN & - \\
\hline 24 & 18,0 & 4,0 & $\mathrm{TN}$ & - \\
\hline
\end{tabular}

Teste de Mann-Whitney

$\mathrm{z}$ calculado $=5,53$

$\mathrm{z}$ críticos $=1,96$

* TN: tiróide normal

- : mapeamento negativo

$+:$ mapeamento positivo

Quando a droga é usada por mais de 60 meses o percentual de disfunção tiroidiana pode duplicar ${ }^{7}$.

Embora o T4 estivesse aumentado em $50 \%$ e $70,8 \%$ dos pacientes nos $3^{\circ}$ e $9^{\circ}$ mês, respectivamente, somente $3(12,5 \%)$ pacientes apresentaram hipertiroidismo clínico, e como se sabe a hipertiroxinemia, nem sempre significa hipertiroidismo ${ }^{13}$. O aumento de $T 4$ as vezes dificulta o diagnóstico. Em nenhum de nossos pacientes com hipertiroidismo a concentração de T3 sérico se elevou. Este resultado diverge dos dados da literatura ${ }^{17}$ e talvez possa ser explicado pela escassa presença em nossa casuística de unicamente três casos de hipertiroidismo. O rT3 sérico, mostrando valores desde 0 início acima da normalidade, limita a importância clínica do rT3 para diagnóstico de hipertiroidismo. A resposta do TSH ao TRH, na nossa casuística, se mostrou ausente ou bloqueada nos casos de hipertiroidismo, servindo como o indicador mais fiel para o diagnóstico de hipertiroidismo. Não encontramos positividade dos anticorpos antitiroglobulina em nenhum dos hipertiroidianos do nosso grupo, reforçando a idéia de que a auto-imunidade provavelmente não seria um fator importante no desenvolvimento do hipertiroidismo ${ }^{818}$. A captação reduzida do iodo radioativo, com cintilografia negativa em $2 / 3$ dos pacientes hipertiroideos está de acordo com dados da literatura ${ }^{8}$. O paciente que apresentou hipercaptação era portador de bócio difuso. Quanto à correlação bócio e hipertiroidismo, somente um paciente por nós submetido a tratamento com amiodarona apresentou bócio difuso $\mathrm{e}$ hiperfunção tiroidiana.

Ao contrário do ocorrido com o hipertiroidismo, nosso achado de $8,3 \%$ de hipotiroidismo é nitidamente inferior ao registrado por outros autores em áreas suficientes de iodo 9101131 34. A disautonomia autonômica, característica da cardiopatia chagásica, pode dificultar o diagnóstico clínico de hipotiroidismo. Por isso o diagnóstico laboratorial de hipotiroidismo em nosso estudo se baseou na elevação da concentração sérica do TSH e redução do T4. O TSH elevado, pode ter sido suficiente para induzir o aparecimento de bócio em um dos nossos casos de hipotiroidismo, provavelmente porque o paciente já apresentava TSH elevado desde os três meses após início do tratamento com amiodarona. Hiper-resposta do TSH ao TRH como preditivo de hipotiroidismo não parece ter sido relevante em nosso estudo. Não observamos positividade de anticorpos em nenhum dos pacientes hipotiroidianos.

Quanto aos níveis inexplicavelmente aumentados do rT3, por nós constatados nos tempos basais iniciais, somente um estudo mais prolongado, de avaliação do rT3 em pacientes chagásicos crônicos sem tratamento com amiodarona, poderia elucidar a questão. Chopra e cols ${ }^{7}$ também encontraram valores aumentados em doenças crônicas não tiroidianas.

Nossos dados indicam que o uso da amiodarona em nossa região deve ser acompanhado de análise 
Barros MAE, Maciel RMB. Estudo prospectivo dos efeitos da amiodarona na funçấo tiroidiana de pacientes chagásicos em área de deficiência de iodo. Revista da Sociedade Brasileira de Medicina Tropical 27:149-155, jul-set, 1994.

dos hormônios tiroidianos e que a função tiroidiana deve ser cuidadosamente monitorizada antes e durante o tratamento com a droga.

\section{SUMMARY}

In order to evaluate the development of thyroid dysfunction during chronic amiodarone treatment in an area deficient in iodine and endemic for Chagas' disease, a group of 24 patients was prospectively studied. Clinical examination and measurement of serum $T 4, T 3, r T 3$, TSH and antithyroglobulin antibodies were performed before and at 3 and 9 months of use of amiodarone. A TSH response 30 minutes after $I$ injection of $200 \mu \mathrm{g}$ of TRH was also compared to TSH basal levels before and during amiodarone treatment. Thyroid radioative uptake and scan were obtained before and nine months after amiodarone was started. Elevated rT3 concentrations were unexpectedly found in two thirds of the patients before treatment. Thyroid dysfunction developed during amiodarone administration in $20,8 \%$ of the patients; $12,5 \%$ became hyperthyroid and $8,3 \%$, hypothyroid (with negative antithyroglobulin antibodies). Positive RAI uptake was seen in one patient with hyperthyroidism and diffuse goiter. Since T3 levels were not found to increase, the diagnosis of amiodarone-related hyperthyroidism was better evidenced by the reduced or blocked TSH response to TRH. Elevated TSH concentration was the best evidence of amiodaroneinduced hypothyroidism. Increase in TSH levels since the beginning of amiodarone therapy may predispose to the growth of a goiter. In conclusion, amiodarone treatment in an iodine deficient area as above should be judiciously decided and thyroid function carefully monitored before and during the use of the drug.

Key-words: Amiodarone. Thyroidfunction. Chagas' disease. lodine deficiency.

\section{REFERÊNCIAS BIBLIOGRÁFICAS}

1. Aguini-Lombardi F, Martino E, Lenziardi M, Grasso L, Pacchiarotti A, Capellini Q, Odoguardi L, Fenzi G, Baschieri L, Pinchera A. Hipertiroidismo iodoindotto da amiodarone. Medicina 76:431-436, 1985.

2. Albert GS, Larry EA, Edward PR. Thyroid dysfunction during chronic amiodarone therapy. Journal of the American College of Cardiology 9:175-183, 1987.

3. Amino N, Hagen SR, Yamada N, Refetoff S. Measurement of circulating thyroid microssomal antibodies by the tanned red cell haemaglutination technique: its usefulness in the diagnosis of autoimmune diseases. Clinical Endocrinology 5:115$125,1976$.

4. Barros MA, Barros AJ, Lopes ER, Vasconcelos JLM, Teodoro CS. Ocorrência das patologias tiroidianas, com especial referêcia às neoplasias, na região do Triângulo Mineiro. Arquivos Brasileiros de Endocrinologia e Metabologia 25:95-98, 1981.

5. Burger $A$, Dinichert $D$, Nicot $P$, Jenny $M$, Lemarchand-Beraud T, Vallotton MB. Effect of amiodarone on serum triiodothyronine, reverse triiodothyronine, thyroxine and thyrotropin. Journal of Clinical Investigation 58:255-259,1976.

6. Burger AG. Effects of certain pharmacologic agents on the peripheral metabolism of thyroxine. In: Ingbar SH, Braverman LE (eds) Werner's-The Thyroid. 5 edition. Lippincott, Philadelfia p.351$360,1986$.

7. Chopra IJ, Solomon DH, Hepner GW, Morgenstein AA, Misleading LY. Low free thyroxine index and usefulness of reverse triiodothyronine measurement in non thyroidal illness. Annals of Internal Medicine 90:905-912, 1979.

8. Fradkin JE, Wolff J. lodine-induced thyrotoxicosis. Medicine 62:1-20, 1983.

9. Heger JJ, Prystowsky EN, Jackamn WM, Naccarelli GW, Warfel KA, Rinkenberger RL, Zipes DP: Amiodarone: clinical efficacy and electrophysiology during long-term therapy for recurrent ventricular tachycardia or ventricular fibrillation. New England Journal of Medicine 305:539, 1981.

10. Heger JJ, Prystowsky EN, Zipes DP. Clinical efficacy of amiodarone in treatment of recurrent ventricular tachycardia and ventricular fibrillation. American Heart Journal 106:887, 1983.

11. Jonckheer MH, Blockx P, Broeckert I, Cornette C, Beckers C. "Low T3 syndrome" in patients chronically treated with an iodine-containing drug, amiodarone. Clinical Endocrinology 9:27-35, 1978.

12. Jorge PT. Enzimaimunoensaio para detecção de anticorpos anti-tiroglobulina no soro. Desenvolvimento metodológico e implicações diagnósticas. Tese de Doutorado, Escola Paulista de Medicina, São Paulo,SP, 1986.

13. Lambert MJ, Burger AG, Galeazzi RL, Engler D. Are selective inereases in serum thyroxine due to indinated inhibitors of $\mathrm{T} 4$ monodeiodination indicative of hiperthyroidism? Journal of Clinical Endocrinology and Metabolism 55:1058-1065, 1982.

14. Lombardi A, Martino A, Braverman LE. Amiodarone and the thyroid. Thyroid Today 2:17, 1990.

15. Lopes ER, Moraes CA, Chapadeiro E, Mineo JR, Leite LC, Guimarães AHB, Rocha A, Gava M. Prevalência da infecção chagásica em necrópsias no 
Barros MAE, Maciel RMB. Estudo prospectivo dos efeitos da amiodarona na função tiroidiana de pacientes chagásicos em área de deficiência de iodo. Revista da Sociedade Brasileira de Medicina Tropical 27:149-155, jul-set, 1994.

Triângulo Mineiro. Revista de Medicina Tropical de São Paulo 26:125-129, 1984.

16. Maciel RMB, Russo EMK, Furlaneto PP. Testes de função tiroideana. In: Guimarães RX, Guerra CCC (eds) Clínica e Laboratório. 3 edition, Sarvier São Paulo p,115-126, 1983.

17. Martino E, Macchia E, Lombardi F, Antonelli A, Lenziardi $M$, Concetti R, Fenzi GF, Baschieri $H$, Pincheră $\mathrm{A}$. Is humoral thy roid autoimmunity relevant in amiodarone iodine-induced thyrotoxicosis? Clinical Endocrinology 24:627-633, 1986a.

18. Martino E, Safran M, Aguini-Lombardi F, Rajatanáven R, Lenziardi M, Fay M, Pacchiarotti A, Aronin N, Macchia H, Haffajee C, Adoguardi L, Love J, Bigalli A, Bascuieri L, Pinchera A, Braverman L. Environmental iodine intake and thyroid dysfunction during chronic amiodarone therapy: Annals of Internal Medicine 101:28-34, 1984.

19. Massin JP, Thomopoulos P, Karan J, Savoie JC. Le risque thyroidien d'un nouveau coronariodilatateur iodé: l'amiodarone (Cordarone). Annales Endocrinologie 32:438-448, 1971.

20. Medeiros-Neto GA, Lobo LCG, Nicolau W. Endemic goiter in Brazil. In: Pan American Health Organization (ed) "Endemic Goiter". World Health Organization John B.Stanbury, New York p. 179182, 1969.

21. Pellon $A B$, Silva W, Borges $P$, Gualberio V. Áreas bocígenas no Brasil. Div Org Sanit, Ministério da Saúde, Brasil, 1956. In: Freire-Maia A, FreireMaia DV (eds) Endemic Goiter and Genetics. Revista Brasileira de Pesquisas Médicas e Biológicas 3:53-58, 1970.

22. Peter T, Hamer A, Mandel W, Weiss D. Evaluation of amiodarone therapy in the treatment of drugresistant cardiac arrhythmias: Long-term followup. American Heart Journal 106:943, 1983.

23. Pritchard DA, Singh BN, Hurley PJ. Effect of amiodarone on thyroid function in patients with ischaemic heart disease. British Heart Journal 37:856860, 1975.

24. Rabinowe DL, Laesen PR, Antman EM George KL, Friedman PL, Jackson RA, Eisenbarth GS. Amiodarone therapy and autoimmune thyroid disease. Increase in a new monoclonal antibody defined T cell subset. American Journal of Medicine
81:53-57, 1986.

25. Russo EMK, Vieira JGH, Maciel RMB, Fonseca RMG. Desenvolvimento e caracterização de métodos de radioimunoensaio para dosagem de iodotironinas. Arquivos Brasileiros de Endocrinologia e Metabologia 26:23-28,1982.

26. Safran M, Fang SL, Bambini G, Pinchera A, Martino E, Braverman L. Effects of amiodarone and desethylamiodarone on pituitary deiodinase actity and thyrotropin secretion in the rat. American Journal of the Medical Sciences 292:136-141, 1986.

27. Sanmarti A, Permanyer GM, Castellanos JM, FozSala M, Galard PM, Soler-Soler J. Chronic administration of amiodarone and thyroid function: a follow-up study. American HeartJournal 108:1262$1268,1984$.

28. Siegel S. Estatística não paramétrica. McGraw-Hill, São Paulo, 1975.

29. Silva JE, Larsen PR. Pituitary nuclear 3,5,3'triiodothyronine and thuyrotropin secretion: an explanation for the effect of thyroxine. Science 198:617-622, 1977.

30. Silva JE, Leonard JL. Regulation of rat cerebrocortical and adenohypophyseal type II 5'deiodinase by thyroxine, triidothyronine and reverse triiodothyronine. Endocrinology 116:1627-1635, 1985.

31. Singh BN, Nademanee K. Amiodarone and thyroid function: Clinical implications during antiarrhythmic therapy. American Heart Journal 106:857, 1983.

32. Smirk TC, Goellner JZ, Brennan MD, Carney JA. Pathology of the thyroid in amiodarone associated thyrotoxicosis. American Journal of Surgical Pathology 11:197-209, 1987.

33. Vagenakis AG, Wang $C$, Burger A, Maloof $F$, Braverman LE, Ingbar SH. Iodine - induced thyrotoxicosis in Boston. New England Journal of Medicine 287:523-527, 1972.

34. Ward DE, Camm AJ, Spurrel RAJ. Clinical antiarrhythmic effects of amiodarone in patients with resistant paroxysmal tachycardias. British Heart Journal 44:82, 1980.

35. Wiersinga WM, Touber JL, Trip M, Van-Royen EA. Uninhibited thyroidal uptake of radioiodine despite iodine excess in amiodarone induced hypothyroidism. Journal Clinical of Endocrinology and Metabolism 63:485-491, 1986. 